\title{
Analysis of the halo background in femtosecond slicing experiments
}

\section{Daniel Schick, Loïc Le Guyader, Niko Pontius, Ilie Radu, Torsten Kachel, Rolf Mitzner, Thomas Zeschke, Christian Schüßler-Langeheine, Alexander Föhlisch and Karsten Holldack}

J. Synchrotron Rad. (2016). 23, 0

\section{IU IUCr Journals CRYSTALLOGRAPHY JOURNALS ONLINE}

Copyright (C) International Union of Crystallography

Author(s) of this paper may load this reprint on their own web site or institutional repository provided that this cover page is retained. Republication of this article or its storage in electronic databases other than as specified above is not permitted without prior permission in writing from the IUCr.

For further information see http://journals.iucr.org/services/authorrights.html 
JOURNAL OF SYNCHROTRON RADIATION

ISSN 1600-5775

Received 4 February 2016

Accepted 9 March 2016

Edited by D. A. Reis, SLAC National Accelerator Laboratory, USA

Keywords: femtosecond slicing; halo; pump-probe; XMCD; X-ray scattering.

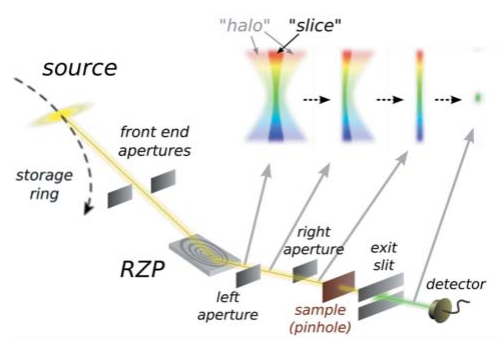

C 2016 International Union of Crystallography

\section{Analysis of the halo background in femtosecond slicing experiments}

\author{
Daniel Schick, ${ }^{a *}$ Loïc Le Guyader, ${ }^{\mathrm{a}, \mathrm{b}}$ Niko Pontius, ${ }^{\mathrm{a}}$ Ilie Radu, ${ }^{\mathrm{a}, \mathrm{c}}$ \\ Torsten Kachel, ${ }^{a}$ Rolf Mitzner, ${ }^{a}$ Thomas Zeschke, ${ }^{a}$ Christian Schüßler-Langeheine, ${ }^{a}$ \\ Alexander Föhlisch ${ }^{a, d}$ and Karsten Holldack ${ }^{a}$ \\ ${ }^{a}$ Helmholtz-Zentrum Berlin für Materialien und Energie GmbH, Albert-Einstein-Strasse 15, 12489 Berlin, Germany, \\ ${ }^{\mathbf{b}}$ European XFEL GmbH, Albert-Einstein-Ring 19, 22761 Hamburg, Germany, 'cMax-Born Institute, Max-Born-Strasse 2A, \\ 12489 Berlin, Germany, and dUniversität Potsdam, Karl-Liebknecht-Strasse 24-25, 14476 Potsdam, Germany. \\ *Correspondence e-mail: daniel.schick@helmholtz-berlin.de
}

The slicing facility FemtoSpeX at BESSY II offers unique opportunities to study photo-induced dynamics on femtosecond time scales by means of X-ray magnetic circular dichroism, resonant and non-resonant X-ray diffraction, and $\mathrm{X}$-ray absorption spectroscopy experiments in the soft X-ray regime. Besides femtosecond X-ray pulses, slicing sources inherently also produce a so-called 'halo' background with a different time structure, polarization and pointing. Here a detailed experimental characterization of the halo radiation is presented, and a method is demonstrated for its correct and unambiguous removal from femtosecond time-resolved data using a special laser triggering scheme as well as analytical models. Examples are given for time-resolved measurements with corresponding halo correction, and errors of the relevant physical quantities caused by either neglecting or by applying a simplified model to describe this background are estimated.

\section{Introduction}

The combination of (soft) X-ray radiation with femtosecond time-resolution has opened up the opportunity to study ultrafast magnetic dynamics as well as the dynamics of photoinduced phase transitions in correlated materials under nonequilibrium conditions with unique sensitivity (Holldack et al., 2010; Radu et al., 2011; Eschenlohr et al., 2013; Kubacka et al., 2014). Element and site specificity allow for accessing individual degrees of freedom in solid state matter on their intrinsic time scales in a selective manner and for studying nano-scale ordering phenomena such as magnetism, charge- and orbitalordering in great detail (Stamm et al., 2007; Beaud et al., 2009). Established experimental techniques used in this context are X-ray absorption spectroscopy (XAS) and X-ray magnetic circular dichroism (XMCD) aiming for spectroscopic and magnetic information, as well as resonant $\mathrm{X}$-ray diffraction (RXRD) combining spectroscopic and structural information.

Within the last 20 years the development of accelerator- and laboratory-based sources of sub-picosecond light pulses from the $\mathrm{THz}$ up to the hard X-ray regime had a huge impact in this field. Alongside free-electron lasers (FELs) (Emma et al., 2010; Patterson et al., 2010), storage-ring-based femtosecond slicing sources (Zholents \& Zolotorev, 1996) have hosted pioneering experiments for the investigation of ultrafast dynamics in solid state matter. The laser slicing of stored relativistic electron bunches (femtoslicing) produces typically $100 \mathrm{fs}$ (FWHM) long X-ray pulses and is currently used for experiments at the Advanced Light Source (Schoenlein et al., 
2000), at the Swiss Light Source (SLS) (Beaud et al., 2007) and at BESSY II (Khan et al., 2006; Brzhezinskaya et al., 2013; Holldack et al., 2014). At the storage ring SOLEIL a new slicing facility is currently being set up (Prigent et al., 2013).

In general, the slicing technique generates femtosecond pulses of tunable photon energies from the hard to the soft $\mathrm{X}$-ray regime with variable polarization which offers great flexibility compared with laboratory-based sources. Currently, only the slicing facility at BESSY II provides femtosecond circularly polarized X-rays allowing for time-resolved XMCD studies of ferro- and ferri-magnetic compounds. Although FELs offer much higher photon flux (Emma et al., 2010), laser slicing remains to date an important alternative for timeresolved X-ray studies. Compared with FEL sources, slicing facilities have the advantage of a higher repetition rate in the $\mathrm{kHz}$ regime, higher stability regarding beam pointing, photon energy and pulse duration, as well as the intrinsic synchronization of laser-pump and X-ray-probe pulses avoiding the need of a complex shot-to-shot correction of temporal jitter (Beye et al., 2012; Harmand et al., 2013).

Since the first experiments at the FemtoSpeX facility at BESSY II (Stamm et al., 2007) an increased laser repetition rate and a new high-transmission refocusing zone plate optics have led to a 120 times increase of the average photon flux (Holldack et al., 2014). The associated drastic increase in sensitivity and data quality of time-resolved experiments allowed for more complex studies requiring a high standard in the quantitative analysis of the measured data. Consequently, a proper quantitative treatment of the inherent so-called 'halo' background radiation turns more and more relevant for the correct interpretation of many experimental data. The halo background radiation superimposes the $100 \mathrm{fs}$ slicing $\mathrm{X}$-ray pulses and is a consequence of the remaining electron bunch excitations from preceding electron-energy-modulation events by the femtoslicing itself. This results in a mainly transversely extended electron bunch portion of the energymodulated electrons generating X-ray radiation with a larger divergence and picosecond time structure after the first few turns (Holldack et al., 2014). Its pulse length at later delays depends on the damping process after the slicing shot and approaches at infinity the measured current bunch length of the undisturbed bunch which at BESSY II is 69 ps (FWHM) for $4 \mathrm{~mA}$ bunch current. This temporal evolution causes the halo background not to be a constant contribution to the slicing signal but to be also delay dependent. The impact this dynamic halo contribution has on the experiment strongly depends on the actual observable of interest as well as the combination of various experimental settings which determine the halo properties. For many experiments, e.g. spectroscopic transmittance studies, the slicing-to-halo ratio can be tuned to a regime with negligible impact of the halo background by adjusting electron beam settings and beamline slits at the cost of total detected X-ray intensity. For other experiments, such intensity loss is not acceptable. The possibility to fully correct for the dynamic halo background would therefore give a higher flexibility in the choice of the experimental settings allowing for more photon-hungry experiments. In this paper, we provide a detailed characterization of the halo background at the FemtoSpeX facility at BESSY II. We present a dedicated laser triggering scheme allowing for an experimental access to the dynamic halo contribution on top of the measured femtosecond slicing traces. We give examples of time-resolved measurements with corresponding halo correction. Finally, we estimate errors of the relevant physical quantities that result from neglecting the halo contribution to the time-resolved traces or by correcting for it using a simplified model.

\section{Generation of slicing and halo X-rays}

To generate femtosecond X-ray pulses by laser slicing at BESSY II, femtosecond near-infrared laser pulses modulate the energy of electrons (up to $\pm 1 \%$ ) within a stored electron bunch in a wiggler (the modulator) (Khan et al., 2006; Holldack et al., 2014). At BESSY II and at the SLS, electron energy dispersive elements following the modulator convert the initial energy modulation into a horizontal angle separation of the core bunch and the energy-modulated portion. Right after the energy modulation and spatial separation, the femtosecond X-ray pulses are harvested in a subsequent undulator (radiator) only $5 \mathrm{~m}$ further downstream, where the energy-modulated portion of the electron bunch emits synchrotron radiation at an angular offset with respect to the core beam and $\sim 100$ fs (FWHM) pulse duration. However, in their subsequent travel along their closed orbit in the storage ring, the energy-modulated electrons are not scraped off from the beam but stay well inside the machine's dynamic acceptance performing betatron and synchrotron oscillations (see the $1 \mathrm{kHz}$ case in Fig. 1). Decoherence effects lead to a randomization, and a stationary isotropic electron bunch halo is formed within a few hundred revolutions after the laser slicing (Streun, 2010). Owing to its incoherence the halo behaves like a beam of enlarged effective emittance, which complicates its separation from the femtosecond component in subsequent femtoslicing events. The effective source size as well as the divergence of the halo fraction are considerably enlarged. From the THz-radiation diagnostics spectra we know that already after a few turns (3-5) the halo pulses quickly stretch longitudinally to the picosecond regime by momentum compaction (Holldack et al., 2006). Whenever these halo electrons pass the radiator again after a few turns they give rise to an X-ray halo with larger opening angle and source size, different photon energy and longer pulse length that still has considerable intensity when the next slicing process occurs. From here on, the term 'halo' always refers to only the halo X-ray radiation, as introduced above.

The magnitude of the halo background radiation is directly linked to the ratio between slicing laser repetition period (typically $166 \mu \mathrm{s}$, corresponding to $6 \mathrm{kHz}$ repetition rate) and the much longer radiation damping time of BESSY II of $8 \mathrm{~ms}$. Fig. 1 compares the temporal evolution of the halo background for three different slicing laser repetition rates. This comparison elucidates the trade-off between increased average femtosecond photon flux and halo background signal 


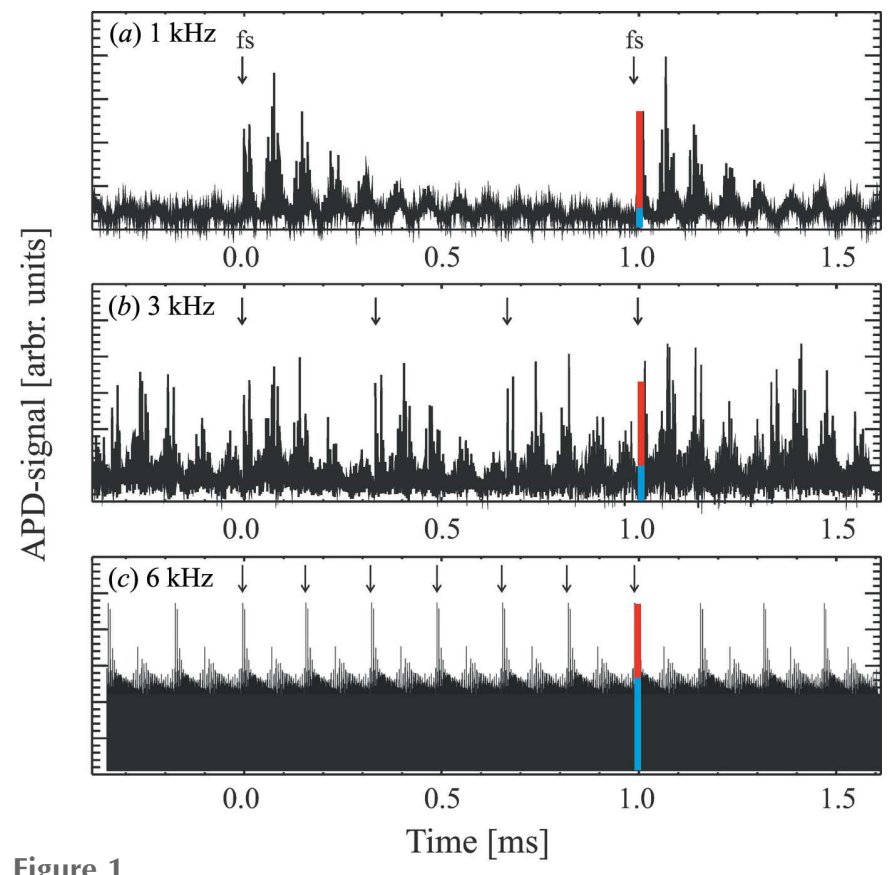

Oscilloscope traces of the X-ray signal (1 mm APD) at the UE56/1 ZPM beamline as a function of time at $850 \mathrm{eV}$ (in $2 \mathrm{eV}$ bandwidth) for three different slicing setups: with (a) $1 \mathrm{kHz},(b) 3 \mathrm{kHz}$ and (c) $6 \mathrm{kHz}$ repetition rate of the laser system. A stationary halo pattern evolves at higher laser frequency and the ratio between the femtosecond signal (red bar) and the halo intensity beneath (blue bar) decreases rapidly approaching a 1:1 ratio at $\sim 6 \mathrm{kHz}$. Operation in the so-called sequence mode is nearly equivalent to the $1 \mathrm{kHz}$ situation (a) with each femtoslicing event occurring every $0.5 \mathrm{~ms}$.

due to higher repetition rate. In turn, due to the rather slow radiation damping, the halo characteristics do not significantly change for several revolutions at a time right before the next slicing process occurs. This can be employed to measure the $\mathrm{X}$-ray halo from these revolutions for a reliable determination of the static and dynamic halo background beneath the sliced $\mathrm{X}$-ray signal as discussed in detail below.

As a way to reduce the halo background at high repetition rates, the so-called 'sequence mode' of the FemtoSpeX facility has been established, alternately slicing three dedicated electron bunches, separated by $12 \mathrm{~ns}^{\mathbf{1}}$ instead of one single electron bunch (see Fig. 5 and Holldack et al., 2014). This effectively reduces the slicing repetition rate for each individual bunch to currently $2 \mathrm{kHz}$ which produces a residual stationary halo close to the $1 \mathrm{kHz}$ case in Fig. 1 . In addition to the standardly used sequence mode, also the undulator parameters (gap, shift), beamline parameters in the ZPM monochromator, and variable apertures at the FemtoSpeX endstation (see §4) have an influence on the slicing-to-halo ratio and can be tweaked for a further reduction of the halo background down to typically $10 \%$ of the overall signal and below. However, the halo background cannot be entirely removed without intensity loss and its influence on the

\footnotetext{
${ }^{1}$ The separation of $12 \mathrm{~ns}$ corresponds to the least common multiple of the ring clock period of $2 \mathrm{~ns}(\widehat{=} 500 \mathrm{MHz})$ and the laser oscillator repetition period of $12 \mathrm{~ns}(\widehat{=} 83.3 \mathrm{MHz})$.
}

experimental results strongly depends on the actual settings and measurement parameters of interest. Especially for very soft X-rays $(<250 \mathrm{eV})$ and elliptical polarization, a proper halo background treatment is mandatory, since the angular separation between slicing and halo components becomes more and more difficult owing to the much larger natural emission opening angles.

\section{Definitions}

Here we introduce the general quantities for the characterization and evaluation of the dynamic halo background contribution. Most of the formulae are common to any generic pump-probe experiment using ultrashort pulsed sources. In order to describe a simplified pump-probe experiment we define the detected intensity $\mathcal{D}(t)$ as the convolution of the sampling pulse $\mathcal{S}(t)$ and the transient response from the sample $\mathcal{R}(t)$ after excitation,

$$
\mathcal{D}(t)=\mathcal{S}(t) * \mathcal{R}(t),
$$

assuming that the laser excitation (pump) is quasi-instantaneous ( $\delta$-like) at $t=0$ and that there is no temporal jitter between pump and probe pulses. An illustration of all terms and definitions is shown in Fig. 2.

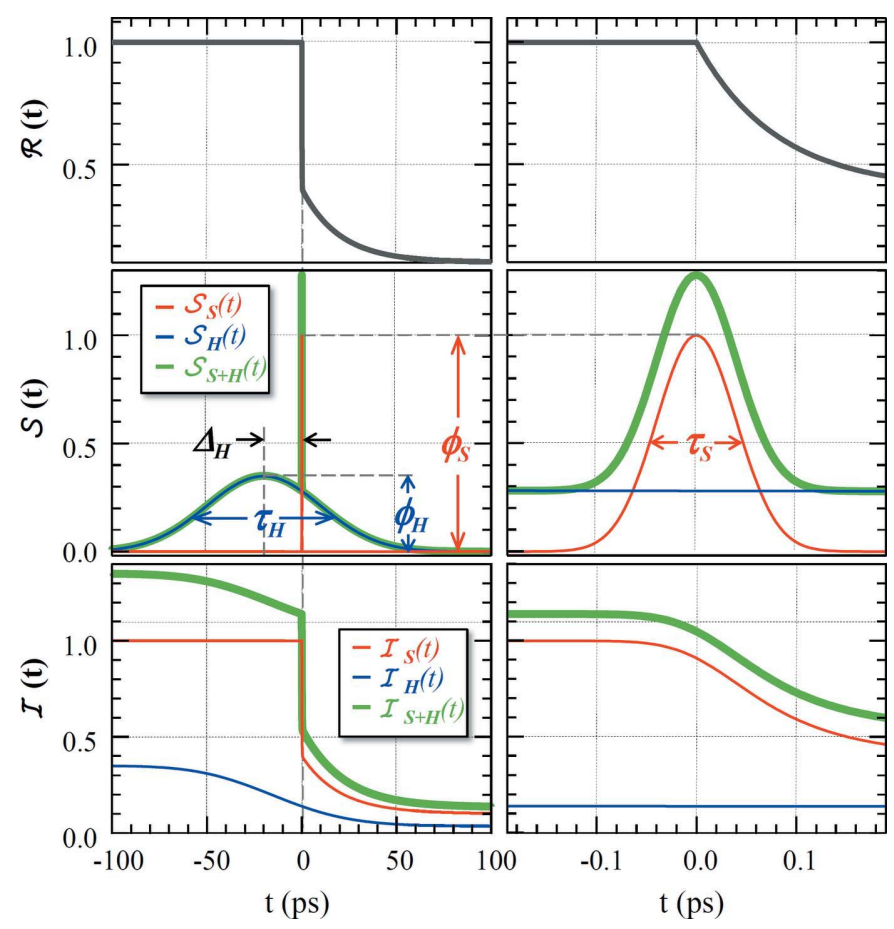

Figure 2

Explanation of the terms and definitions used in $\S 3$. Left and right columns represent the different time ranges of the halo background and femtosecond sliced X-rays, respectively. The top plots show a typical dynamic response $\mathcal{R}(t)$ (dark gray) of a model sample upon laser-pump. The center plots show the time structure $\mathcal{S}(t)$ of the femtosecond sliced (red) and the halo background (blue) X-ray pulses. The green line is the sum of both contributions. The bottom plots show the corresponding signals $\mathcal{I}(t)$ as detected in a model experiment. Clearly, due to the presence of the halo background, the green line shows quantitative and qualitative deviations when directly compared with the sample response function $\mathcal{R}(t)$. 
The sampling pulse $\mathcal{S}(t)$ is described by a normalized Gaussian temporal profile $g(t)$ and the photon flux $\varphi$ as

$$
\mathcal{S}(t)=\varphi g(t)=\frac{\varphi}{\sqrt{2 \pi} \sigma} \exp \left[-\frac{\left(t-t_{0}\right)^{2}}{2 \sigma^{2}}\right],
$$

where $\sigma$ is the width of the Gaussian $(\sigma \simeq \mathrm{FWHM} / 2.35)$ and $t_{0}$ is the delay of the pulse, which is in general set to $t_{0}=0$. For simplicity we assume that without pumping the sample is perfectly transparent or a perfect mirror, depending on the experimental geometry. The transient response of the sample is therefore unity before excitation, $\mathcal{R}(t \ll 0)=1$, and thus the unpumped intensity is time-independent and given by

$$
\mathcal{D}_{0}=\mathcal{D}(t \ll 0)=\varphi .
$$

As for most time-resolved pump-probe experiments only changes in the relative intensity, $\mathcal{I}$, are of interest. We can define

$$
\mathcal{I}(t)=\frac{\mathcal{D}(t)}{\mathcal{D}_{0}}=g(t) * \mathcal{R}(t),
$$

which is independent of the incident photon flux $\varphi$.

For a realistic experiment at the FemtoSpeX facility we have to account for the picosecond halo background in addition to the femtosecond sliced X-rays pulses. Accordingly, we define the photon flux $\varphi_{\mathrm{S}}$ and $\varphi_{\mathrm{H}}$ for the sliced and halo contributions, respectively. Similarly, we can define the temporal profiles for $g_{\mathrm{S}}(t)$ and $g_{\mathrm{H}}(t)$ which have obviously different widths $\sigma_{\mathrm{S}}$ and $\sigma_{\mathrm{H}}$ and can be also delayed in time with respect to each other. By defining the slicing fraction

$$
\beta=\frac{\varphi_{\mathrm{S}}}{\varphi_{\mathrm{S}}+\varphi_{\mathrm{H}}}=\frac{\varphi_{\mathrm{S}}}{\varphi_{\mathrm{S}+\mathrm{H}}},
$$

where the index $\mathrm{S}+\mathrm{H}$ generally represents the sum of the slicing and halo contribution, we can write the detected relative intensity as follows,

$$
\mathcal{I}_{\mathrm{S}+\mathrm{H}}(t)=\beta g_{\mathrm{S}}(t) * \mathcal{R}_{\mathrm{S}}(t)+(1-\beta) g_{\mathrm{H}}(t) * \mathcal{R}_{\mathrm{H}}(t) .
$$

We point out that the transient sample response $\mathcal{R}(t)$ probed by the sliced and halo X-rays is not necessarily identical. Slicing and halo photons have a different spatial profile on the sample such that the probed sample region for both contributions might be differently excited (see $\$ 5.1$ ).

\subsection{X-ray magnetic circular dichroism}

X-ray magnetic circular dichroism (XMCD) allows for probing the magnetic order in ferro- and ferri-magnetic materials and alloys in an element-specific manner (Stöhr \& Siegmann, 2006; Carva et al., 2009). Generally, XMCD is defined as the difference of a material's absorption coefficient when measured with the two opposite helicities of a fully circularly polarized X-ray beam in a fixed geometry. The most direct way to measure the XMCD of a ferro-/ferrimagnetic sample is in transmission geometry. Here the transmitted intensity depends on the magnitude and sign (+ or - ) of the projection of the magnetization onto the X-ray helicity vector as

$$
T^{ \pm}=\varphi \exp \left[-\mu^{ \pm}\right]
$$

In this notation $\mu^{ \pm}$depends on the degree of the X-ray circularity as well as the angle between the magnetization and the X-ray photon helicity vectors. In good approximation ${ }^{2}$ and for practical reasons we can introduce the dependence of $\mu^{ \pm}$ on the sample macroscopic magnetization $m$ as

$$
\mu^{ \pm}=\mu_{0} \mp \frac{\alpha}{2} m \text {. }
$$

Here, the sample magnetization is limited to the range $-1 \leq m \leq 1$ where +1 and -1 correspond to opposite directions of maximum (saturated) magnetization and $m=0$ to the absence of any detectable magnetic order. The parameter $\alpha$ specifies the dependence on the sample thickness, the degree of X-ray helicity, and the angle between the magnetization and the X-ray photon wavevectors. The material absorption in absence of any macroscopic magnetization is denoted as $\mu_{0}$. In this work we define the XMCD intensity $\chi$ as

$$
\chi=\ln T^{+}-\ln T^{-}=\mu^{+}-\mu^{-}=\alpha m .
$$

For all time-resolved measurements of ultrafast magnetic dynamics we assume $m(t \ll 0)=1$ for the initial state of the sample. Accordingly, the unpumped XMCD $\chi_{0}=\chi(t \ll 0)$ gives direct access to the XMCD amplitude $\alpha$.

In a time-resolved XMCD experiment we can rewrite equation (7) again as a convolution with a Gaussian sampling pulse,

$$
T^{ \pm}(t)=\varphi g(t) * \exp \left[-\mu^{ \pm}(t)\right]
$$

leading to the transient XMCD which probes the timedependent magnetization $m(t)$,

$$
\begin{aligned}
\chi(t)= & \ln \left\{g(t) * \exp \left[\frac{\alpha}{2} m(t)\right]\right\} \\
& -\ln \left\{g(t) * \exp \left[-\frac{\alpha}{2} m(t)\right]\right\} .
\end{aligned}
$$

Note that

$$
\chi(t) \neq g(t) * \alpha m(t),
$$

since the natural logarithm and the convolution do not commute. For a realistic XMCD slicing experiment at the FemtoSpeX facility we again have to account for the halo background, as follows,

$$
\begin{aligned}
\chi_{\mathrm{S}+\mathrm{H}}(t)= & \ln \left\{\beta g_{\mathrm{S}}(t) * \exp \left[\frac{\alpha_{\mathrm{S}}}{2} m_{\mathrm{S}}(t)\right]\right. \\
& \left.+(1-\beta) g_{\mathrm{H}}(t) * \exp \left[\frac{\alpha_{\mathrm{H}}}{2} m_{\mathrm{H}}(t)\right]\right\} \\
& -\ln \left\{\beta g_{\mathrm{S}}(t) * \exp \left[-\frac{\alpha_{\mathrm{S}}}{2} m_{\mathrm{S}}(t)\right]\right. \\
& \left.+(1-\beta) g_{\mathrm{H}}(t) * \exp \left[\frac{\alpha_{\mathrm{H}}}{2} m_{\mathrm{H}}(t)\right]\right\},
\end{aligned}
$$

where $\alpha_{\mathrm{S}}$ and $\alpha_{\mathrm{H}}$ are the XMCD amplitudes of the sliced and halo X-rays, respectively. As we will see later on, the two latter parameters usually have opposite sign, since the halo and

\footnotetext{
2 This holds when the anisotropy induced by the magnetization has only negligible effect on the optical matrix-elements, i.e. when the X-ray magnetic linear dichroism (XMLD) is negligible, which is mostly the case.
} 
sliced X-rays have opposite helicity (see §5.3). Moreover, the laser-induced magnetization dynamics $m_{\mathrm{S}}(t)$ and $m_{\mathrm{H}}(t)$ do not necessarily have to be identical (see §5.1).

In order to determine the slicing fraction $\beta$ for an XMCD transmission experiment, one needs to define the intermediate quantity $X^{ \pm}$from the unpumped/static transmitted intensities as follows,

$$
X^{ \pm}=\frac{T_{0, \mathrm{H}}^{ \pm}}{T_{0, \mathrm{~S}}^{ \pm}}=\frac{1-\beta}{\beta} \exp \left[\frac{1}{2}\left( \pm \alpha_{\mathrm{H}} \mp \alpha_{\mathrm{S}}\right)\right] .
$$

Then $\beta$ can be derived from the product of $X^{+}$and $X^{-}$by

$$
\beta=\frac{1}{1+\sqrt{X^{+} X^{-}}} .
$$

\section{Beamline and endstation setup}

The experimental setup at the UE56/1 ZPM beamline of the FemtoSpeX facility at BESSY II has been recently described in detail (Holldack et al., 2014). The characterization of the PGM beamline at UE56/1 regarding the halo background is analogue and has been omitted for clarity. In short, the ZPM beamline comprises one single optical element, a reflection zone plate which combines both horizontal focusing as well as energy dispersion and focusing along the vertical axes (compare Fig. 3). Photon energy resolution up to $E / \Delta E=500$ can be achieved by selecting the desired photon energy by a vertical slit $(20-100 \mu \mathrm{m})$ after transmitting (diffraction or

\begin{tabular}{|c|c|c|c|c|c|c|c|}
\hline element & 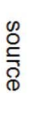 & 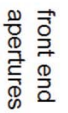 & $\underset{N}{\mathbb{N}}$ & 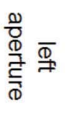 & 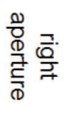 & $\begin{array}{l}\frac{\partial}{3} \cdot \frac{\infty}{3} \\
\frac{0}{3} \\
\frac{0}{D} \frac{0}{D}\end{array}$ & 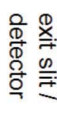 \\
\hline $\begin{array}{l}\text { Distance } \\
\text { (m) }\end{array}$ & 0 & 11.3 & 26.5 & 30.5 & 31.4 & 31.5 & 31.9 \\
\hline
\end{tabular}

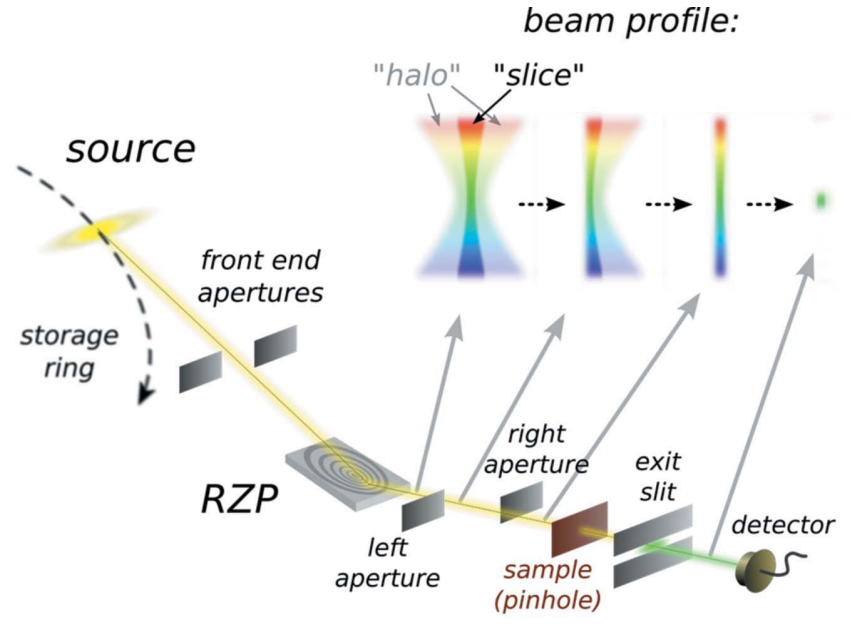

Figure 3

Scheme of the UE56/1 ZPM beamline of the FemtoSpeX facility at BESSY II. The X-ray beam (yellow) is shaped by apertures, slits and pinholes. The reflecting zone plate (RZP) is used for focusing and dispersing the X-rays vertically. The table displays the distances of all beamline components along the X-ray path. reflection from) the sample in front of the detector. Apertures located in the beamline suppress the core beam radiation as well as most of the halo background and primarily transmit the sliced femtosecond X-ray pulses. Details of the spatial characteristics of the halo background and the femtosecond X-ray radiation indicated in Fig. 3 will be discussed in $\$ 5.1$.

\subsection{Data acquisition and laser triggering scheme}

In a time-resolved experiment any time-dependent background contribution synchronized to the femtosecondprobing pulse is as well affected by the intrinsic dynamics of the probed sample. In order to correctly remove the delaydependent dynamic halo signal from time-resolved traces we have implemented a dedicated triggering setup for the laser system and the data acquisition (DAQ) at the FemtoSpeX facility. The corresponding timing scheme including the X-ray signal, pump laser pulses and the DAQ timing are presented in Fig. 4 and explained in the following sections.

The dynamic halo contribution to the femtosecond timeresolved X-ray traces is directly measured by synchronizing pump-laser and DAQ to the dedicated slicing electron bunches three revolutions before the subsequent slicing

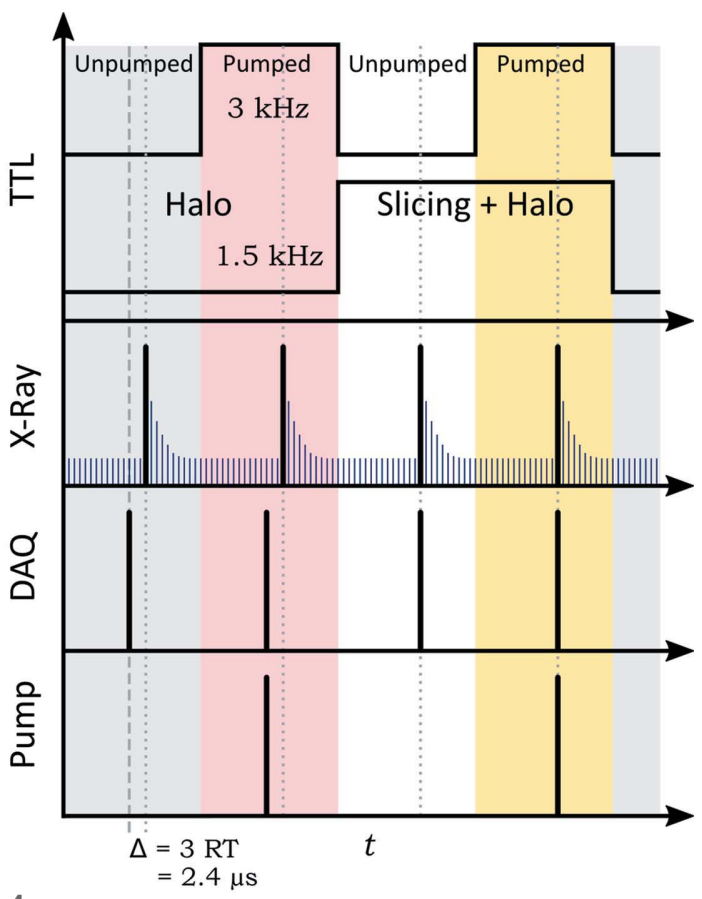

Figure 4

The two nested TTL signals (top) of 3 and $1.5 \mathrm{kHz}$, respectively, establish measurement of the four possible combinations of acquiring unpumped or pumped and halo or (slicing + halo) signals. The simplified X-ray signal (at $6 \mathrm{kHz}$ ) consists of a tall bold black bar representing the signal from the laser sliced bunches (slicing + halo) and small blue bars representing the remaining halo radiation for the subsequent storage ring revolutions. The data acquisition (DAQ) is triggered twice three round trips (RT) before ( $-2400 \mathrm{~ns})$, and twice simultaneously with the actual laser slicing event. The former acquisitions yield the pure unpumped and pumped halo signals while the latter provide the unpumped and pumped slicing + halo signals, respectively. The pump laser amplifier (bottom) alternatingly generates one pump pulse in two laser slicing events, corresponding to $3 \mathrm{kHz}$ repetition rate but with the same timing as the DAQ. 
process occurs. This temporal shift of $-2400 \mathrm{~ns}$ is derived as the least common multiple of the storage-ring revolution period of $800 \mathrm{~ns}(\widehat{=} 1.25 \mathrm{MHz})$ and the laser oscillator period of $12 \mathrm{~ns}$ ( $\widehat{=} 83.3 \mathrm{MHz}$ ). As discussed above in $\S 2$, the halo signal recorded for this revolution is in good approximation identical to that contained in the femtosecond X-ray signal (slicing + halo). Accordingly, the static/unpumped halo contribution is measured for the same revolution but without the pump-laser photo-exciting the sample. The triggering scheme finally combines the measurement of the static and dynamic halo X-ray background with the acquisition of the femtosecond pumped and unpumped X-ray traces (i.e. slicing + halo), respectively, in a quasi-simultaneous fashion: all four signals are acquired by two nested sequencing steps, one alternating between the pumped and unpumped case by a $3 \mathrm{kHz}$ frequency, and the other changing between the sliced revolution and that three revolutions before by a frequency of $1.5 \mathrm{kHz}$.

The experimental implementation of this triggering scheme is based on a field-programmable gate array (FPGA) delay generator (BME_SG08p, Bergmann Messgeräte Entwicklung $\mathrm{KG}$ ) and is shown in Fig. 5. We use two Ti:sapphire amplifiers (Legend Elite Duo, COHERENT) which are intrinsically synchronized by being seeded by a single Ti:sapphire oscillator (Micra, COHERENT). The laser oscillator (83.3 MHz $\widehat{=}$ $12 \mathrm{~ns}$ ) is phase-locked to the $500 \mathrm{MHz}$ ( $\widehat{=} 2 \mathrm{~ns}$ ) master clock of the BESSY II storage ring. The first laser amplifier provides $800 \mathrm{~nm}$ wavelength femtosecond laser pulses at $6 \mathrm{kHz}$ repetition rate ( $\widehat{=} 166.7 \mu \mathrm{s}$ ) for the electron bunch slicing. The second laser amplifier generates the femtosecond pulses for sample excitation. It is operated at half the slicing frequency, i.e. $3 \mathrm{kHz}$ repetition rate ( $\widehat{=} 333.3 \mu \mathrm{s}$ ), allowing for alternately acquiring the pumped and unpumped signal.

The X-ray detection at the beamline endstation is routinely carried out by a fast avalanche photodiode (APD; LaserComponent SAR3000) operated close to the breakthrough voltage in single-photon-counting mode. Here, the preamplified X-ray signal from the APD is recorded with a slicing repetition rate of $6 \mathrm{kHz}$ by a combination of constant fraction discriminators and logic gates which sort each shot into one of four counters (994 Counter, ORTEC) according to the nested 1.5 and $3 \mathrm{kHz}$ TTL signals which mark each pulse as pumped or unpumped and halo or slicing + halo. Currently, the triggering scheme, as shown in Fig. 4, is not available for the APD's proportionality mode where a boxcar averager and an oscilloscope are used to record the data. We plan to overcome this limitation with a state-of-the-art FPGA-based digitizer which can handle the separation of the four different pulse markers at the full repetition rate of $6 \mathrm{kHz}$ without dead-time. Until then, boxcar and oscilloscope measurements have to be carried out in a step-by-step mode, where the delay of the pump-laser amplifier is only alternated once per time step.

\section{Halo characteristics}

Since the X-rays emitted from halo electrons have passed the magnetic lattice of the storage ring many times, they evolve different properties compared with the femtosecond sliced photons: (i) halo photons are different in photon energy and bandwidth; (ii) the transverse effective source size and their divergence is larger; (iii) their pulse shape and temporal pattern as well as (iv) the polarization (helicity) of halo photons is different. The detailed characterization of these properties is not only mandatory for estimating the relevance of the halo contribution to femtosecond pump-probe experiments at the FemtoSpeX facility but also for experimentally reducing the halo background as much as possible.

\subsection{Spatial profile}

The source of the halo has a larger effective lateral distribution and divergence than the source of the regular or the femtosecond sliced X-rays. By the reflection zone plate the $\mathrm{X}$-ray source region is mapped (demagnified by a factor of 5.2) onto the exit slit of the ZPM beamline and simultaneously vertically energy-dispersed (compare Fig. 3).

The green traces in Figs. 6(a) and 6(c) were recorded with the detection synchronized to the sliced X-ray pulses. This signal contains in addition to the pure femtosecond X-ray contribution also the halo background and a minor portion of the regular core-bunch (here called multibunch). The blue traces were taken with the detection synchronized to the storage ring revolution before the sliced revolution and comprises the halo background as well as a small portion of

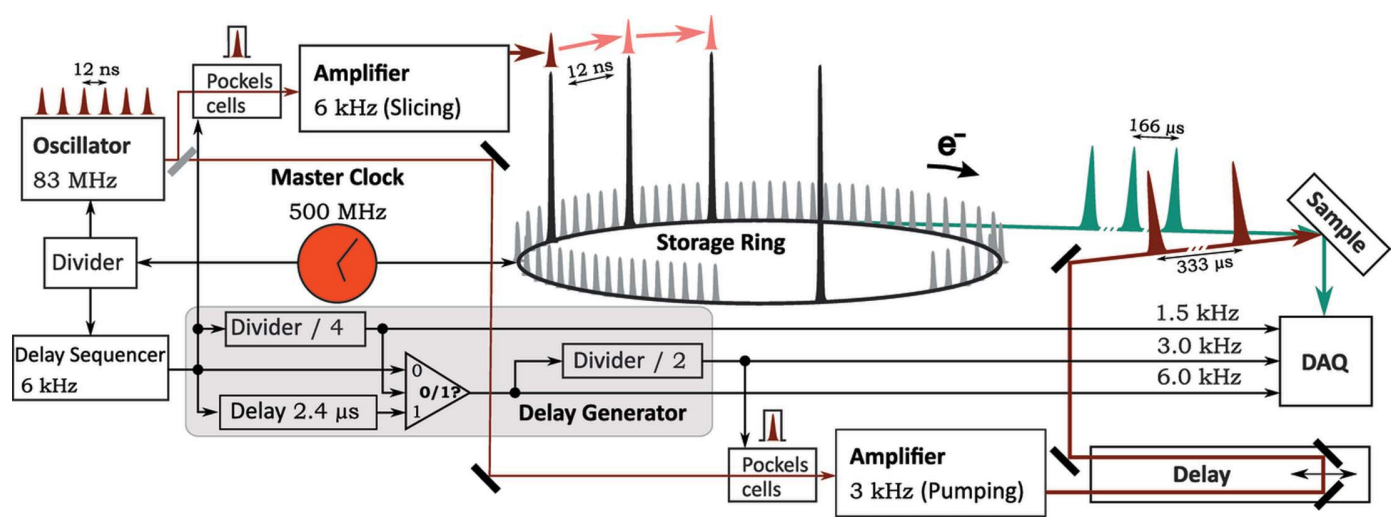

Figure 5

Triggering setup for operating the femtosecond laser system and the DAQ together with the BESSY II storage ring (see text and compare also Fig. 4). 

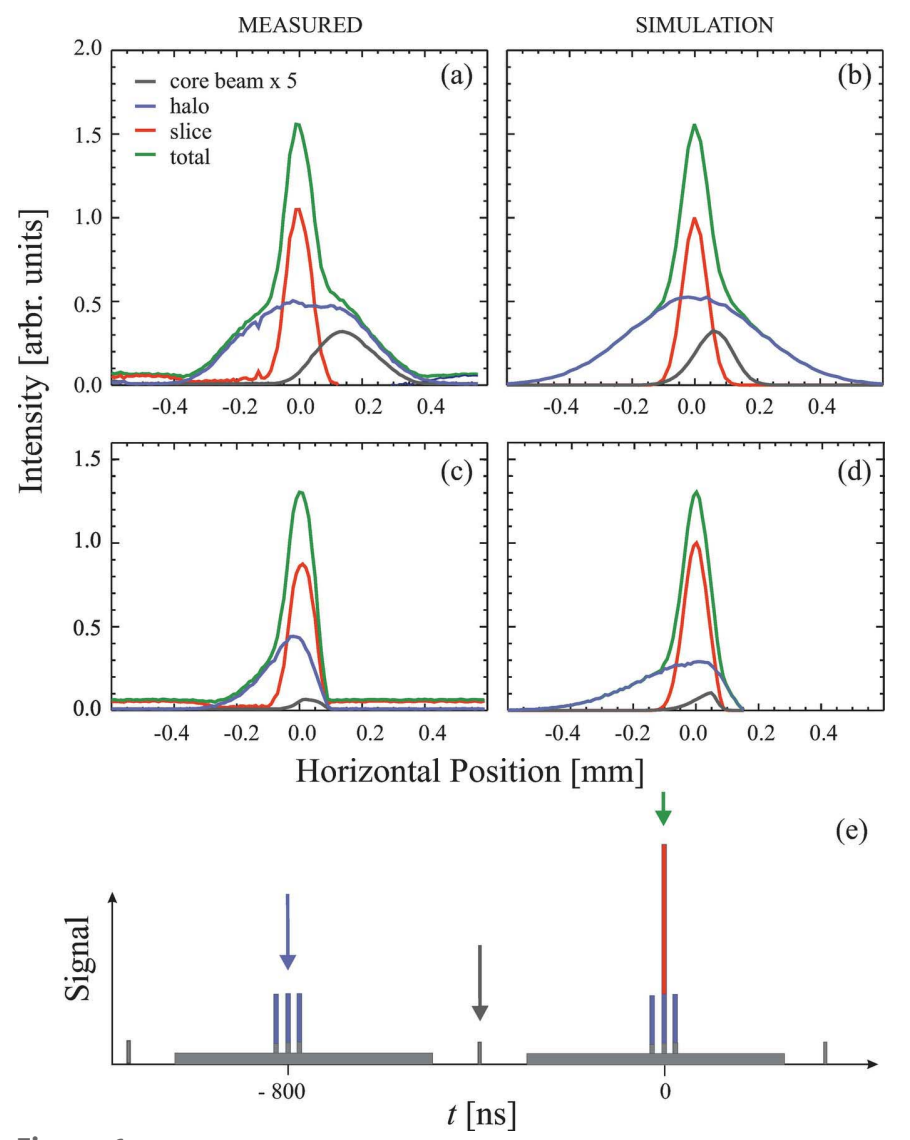

Figure 6

Measured $(a, c)$ and simulated $(b, d)$ horizontal intensity distributions of the various components of the femtosecond X-rays at the sample position of the ZPM beamline. The measurements were performed at $710 \mathrm{eV}$ photon energy. The bottom sketch $(e)$ schematically shows the APD timetrace of the revolution where the electron bunch slicing occurs and the one before $(-800 \mathrm{~ns})$ allowing the background to be measured. The arrows indicate the times at which the APD signal was recorded to probe according contributions. The color code is as follows: green corresponds to the total intensity associated with the sliced revolution; blue corresponds to the background intensity associated with the preceding revolution; dark grey is the remaining intensity of the core bunch which experimentally was recorded on the bunch in the hybrid gap; red indicates the deduced pure femtosecond X-ray signal. The top plots $(a, b)$ show the intensity distributions with full acceptance of the ZPM beamline. In $(c, d)$ the background contribution is reduced using a knife-edge from the right (see text).

the multibunch background. The latter can be measured with the detection synchronized to one of the multibunch bunches (dark grey). The pure femtosecond X-ray contribution can be derived from these measured traces by subtracting the contribution from the revolution before the sliced bunch from that measured when synchronized to the femtosecond X-ray pulse resulting in the red trace.

It can be seen that the pure femtosecond radiation component has a narrow distribution of $\sim 100 \mu \mathrm{m}$ width. This is due to the fact that the femtosecond X-ray photons stem from electrons of a rather well defined energy $(1.72 \mathrm{GeV}$, $-1 \%)$ and small source size ( $\sigma=0.16 \mu \mathrm{m}$ determined from the simulations below) basically generating X-ray radiation on the undulator axes of the radiator with accordingly low divergence $\left(\sigma^{\prime}=20 \mu \mathrm{rad}\right)$. In contrast, the spatial distribution of the halo background is as broad as $\sim 400 \mu \mathrm{m}$ which results from the initially comparatively broad electron energy spread $( \pm 1 \%)$ as well as transverse distributions and the accordingly extended lateral electron bunch size and larger emittance. The multibunch contribution finally originates from the, by $\sim-0.5$ to $-0.8 \mathrm{~m}$ rad deflected, core electron beam which has the regular electron storage ring energy and low emittance ( $5 \mathrm{~nm} \mathrm{rad}$ ) but with a spatial offset to the on-axis beam due to non-vanishing dispersion in the slicing section (Khan et al., 2006).

Based on the source parameters for a BESSY II high-beta straight section, in which the radiator of the femtoslicing source is located, we carried out a simulation of the halo and femtosecond X-ray beam components propagating down the ZPM beamline up to the exit slit and finally resulting in the horizontal spatial profiles in the focal point. Such a simulation was performed by combinations of the codes WAVE and RAY (Scheer, 2012; Schäfers, 2008). Results of the simulations are plotted in Figs. 6(b) and 6(d). The size and divergence of the regular and the femtosecond beams are used as input parameters, while the source size of the halo beam was used as the only fit parameter. An excellent agreement of measurement and simulation of the horizontal spot profiles of the various components can be achieved.

The partial spatial separation of the femtosecond and halo $\mathrm{X}$-rays allows for a reduction of the halo contribution using horizontal knife-edges. This is shown in Fig. 6(c). These spatial spot distributions were recorded suppressing the background by a knife-edge on the right-hand side and the left-hand side of the beam $100 \mathrm{~mm}$ and $830 \mathrm{~mm}$ upstream of the focus, respectively. Due to the larger distance of the left-hand-side knife-edge from the focal point the cut-off is somewhat smeared out (half-shadow effect). The effect of the knife-edge is also nicely reproduced in the simulation shown in Fig. $6(d)$. Obviously, with use of the knife-edges it is possible to reduce the core bunch contribution to a negligible portion. For this reason we will continue the discussion without considering the core bunch background and focus on the halo background only.

Note that the dissimilar halo and femtosecond X-ray spatial profiles (even when using knife-edges) lead to the fact that both contributions to some extent (a few $10 \mu \mathrm{m}$ ) probe different areas of the sample. The reason for this is their source displacement as mentioned above. Together with a spatially varying pump-laser fluence this can lead to different excitation conditions for both contributions. Therefore $\mathcal{R}_{\mathrm{H}}(t)$ $\left[m_{\mathrm{H}}(t)\right]$ is not necessarily identical to $\mathcal{R}_{\mathrm{S}}(t)\left[m_{\mathrm{S}}(t)\right]$ in equations (6) and (13).

\subsection{Temporal profile}

The temporal profile of the halo background pulses is determined by the momentum compaction of the laser-excited electrons in the sliced bunch after approximately 600 revolutions in the storage ring. Numerical simulations (Kuske, 2011; Streun, 2010) point to a duration of several tens of picoseconds of the halo pulses which in addition can be delayed 
and asymmetric in time. After introduction of terms and a general discussion of the sliced and halo X-ray time-structures in a pump-probe measurement in $\S 3$, we will now turn to the data treatment in specific experimental examples, where the individual sliced and halo signals $\mathcal{D}_{\mathrm{S}}(t)$ and $\mathcal{D}_{\mathrm{H}}(t)$ contributions have been measured as described in $\$ 4.1$. We conducted a representative pump-probe experiment which probes the antiferromagnetic order in metallic dysprosium using linear polarized resonant soft X-ray diffraction at the Dy $M_{5}$-edge $(\sim 1292 \mathrm{eV})$ similar to the experiment described by Holldack et al. (2010). The intensity of the probed magnetic $(00 \tau)$ peak with $\tau \simeq 0.18$ reciprocal lattice units is a measure of the antiferromagnetic order in the sample [see Ott et al. (2006) for details]. The photo-excitation thus leads to a fast decrease of this magnetic order beginning at 0 ps time delay, as shown in Fig. 7(a).

The detected halo $\mathcal{D}_{\mathrm{H}}(t)$ (blue symbols) and slicing + halo $\mathcal{D}_{\mathrm{S}+\mathrm{H}}(t)$ (green symbols) data have been directly measured whereas the pure slicing data (red symbols) represent the difference of the absolute intensities of the two former transients $\mathcal{D}_{\mathrm{S}}(t)=\mathcal{D}_{\mathrm{S}+\mathrm{H}}(t)-\mathcal{D}_{\mathrm{H}}(t)$. We describe the photo-induced dynamics in the sample $\mathcal{R}(t)$ by a double-exponential decay function,

$$
\begin{aligned}
\mathcal{R}(t)= & A_{1} H(t)\left[\exp \left(-t / \tau_{1}\right)-1\right] \\
& +A_{2} H(t)\left[\exp \left(-t / \tau_{2}\right)-1\right]+1,
\end{aligned}
$$

where $H(t)$ represents the Heaviside step function. The convolution of $\mathcal{R}(t)$ with a 100 fs (FWHM) Gaussian pulse profile $g_{S}(t)$ is then used for fitting the extracted slicing data $\mathcal{I}_{\mathrm{S}}(t)=\mathcal{D}_{\mathrm{S}}(t) / \mathcal{D}_{0, \mathrm{~S}}$ [red line in Fig. $7(b)$; see $\S 3$ for details $]$. The fit determines the exact temporal overlap of the laser pump and slicing X-ray probe pulses which is set to $t_{0, \mathrm{~S}}=0 \mathrm{ps}$. The fast time constant of the decay is fitted to $\tau_{1}=0.15 \mathrm{ps}$ with a relative amplitude of $A_{1, S}=0.15$, and the slower time constant to $\tau_{2}=16.83$ ps with a relative amplitude of $A_{2, \mathrm{~s}}=0.69$. The total change of intensity is thus $A_{\text {tot, } \mathrm{S}}=A_{1}+A_{2}=0.84$.

In order to determine the temporal profile of the halo pulses we fit the according data $\mathcal{I}_{\mathrm{H}}(t)$ (blue symbols) in Fig. 7(b) with the same fit function as before but we fix all but one parameters of the sample dynamics $\mathcal{R}(t)$ to the values above. The parameter left free is the total intensity change $A_{\text {tot }}$ since the halo potentially samples a differently excited sample region with respect to the sliced X-rays as explained above. However, the ratio $A_{1} / A_{2}$ is kept constant assuming that both driven processes are proportional to the exciting pump fluence. In addition, we allow for different pulse length and arrival time of the halo pulses $g_{\mathrm{H}}(t)$ with respect to the sliced $\mathrm{X}$-rays $g_{\mathrm{S}}(t)$. The result of this fit determines the halo pulse length to $\sigma_{\mathrm{H}}^{\mathrm{FWHM}}=144 \mathrm{ps}$ and a delay of approximately $t_{0, \mathrm{H}}=$ 24 ps between the halo and sliced X-rays. The total change of intensity for probing only with the halo pulses is $A_{\text {tot, } \mathrm{H}}=0.66$ which is significantly less than for the sliced X-rays. This difference can be directly linked to the different spatial profiles of the halo beam which is larger than the slicing beam and thus additionally probes an effectively less excited sample area. The fit of the slicing + halo data $\mathcal{I}_{\mathrm{S}+\mathrm{H}}(t)$ (green symbols)
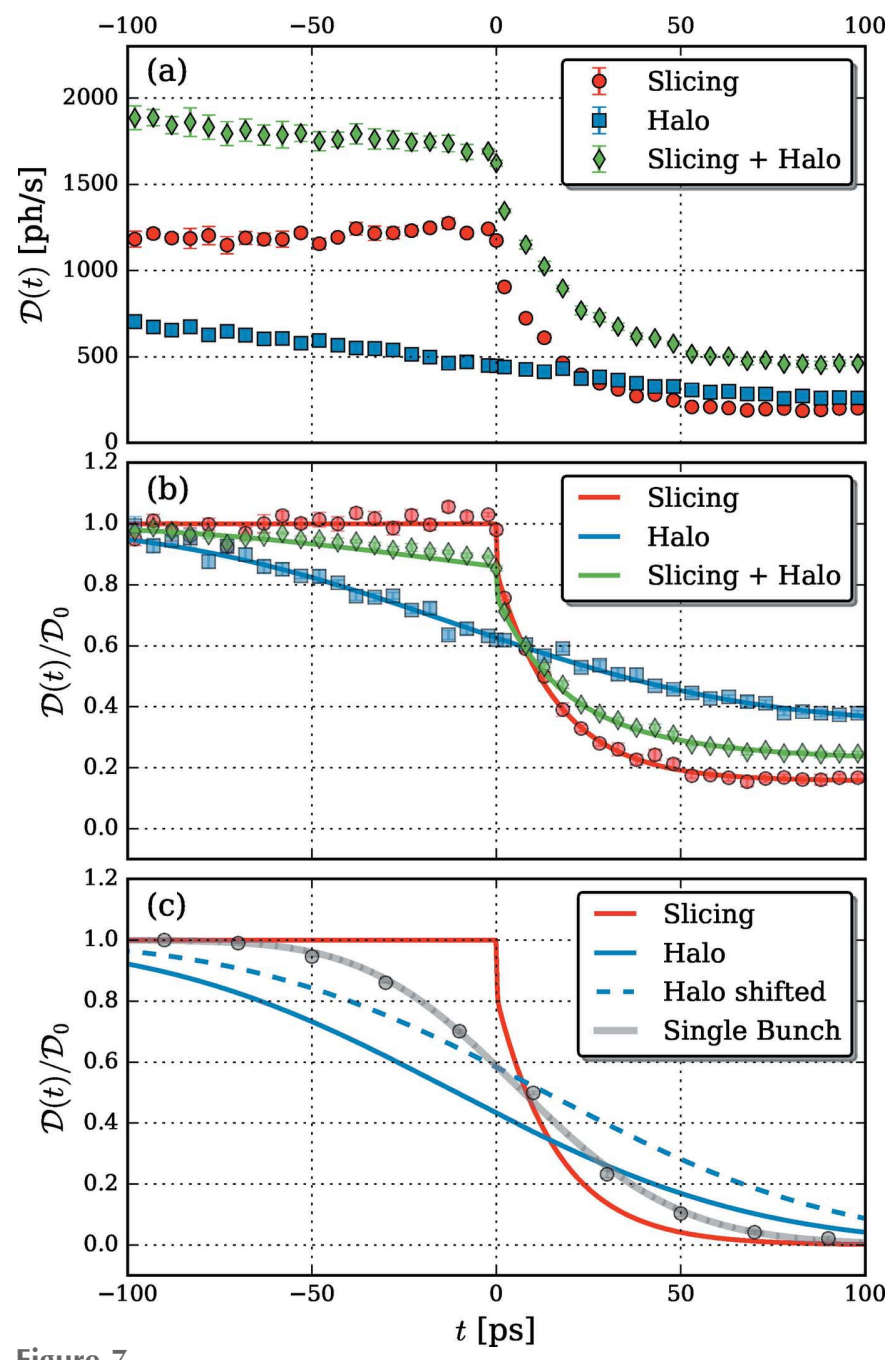

Figure 7

(a) Time-resolved resonant soft X-ray diffraction measurement at the Dy $M_{5}$-edge $(1292 \mathrm{eV})$. (b) Same data as in (a) but representing the relative change. The symbols represent the experimental data and the solid lines the corresponding fits; see text. The pure slicing contribution (red) is determined by subtracting the halo (blue) from the original slicing + halo (green) data. The slicing fraction of the signal is $\beta=0.625$. Note that the subtraction is performed with absolute and not relative data. (c) All relative data are scaled in amplitude to $100 \%$ intensity change for better comparison. The slicing (red) and halo (blue) fits are the same as in $(b)$. The blue dashed line represents the halo fit but shifted to the same time zero as for the slicing. The gray data and fit show the same dynamics but probed with the single bunch of BESSY II.

is the weighted average of the slicing fit (red line) and the halo fit (blue line), as described by equation (6). The weighting is given by the slicing fraction of $\beta=0.625$ and the halo fraction $(1-\beta)=0.375$.

In Fig. 7(c) we show in addition to the slicing and halo fits from Fig. 7(b) the sample dynamics probed with the conventional single bunch of BESSY II in the hybrid mode (gray symbols) with all transients scaled to $A_{\text {tot }}=1$ for better comparison. The blue dashed line represents the halo fit from Fig. 7(b) but shifted to the arrival time of the sliced X-rays $t_{0, \mathrm{H}}=0$. We perform the same fitting procedure for the regular single-bunch data as above for the halo and obtain a pulse length of approximately $\sigma_{\text {single }}^{\mathrm{FWHM}}=72 \mathrm{ps}$ and a delay of $t_{0, \text { single }}=$ 
5 ps for the single bunch compared with the sliced X-rays. This small delay is mainly due to electronic synchronization issues. These measurements show that the halo pulses are significantly longer and delayed in time with respect to the singlebunch time structure. The extracted pulse parameters are only valid assuming a Gaussian temporal shape of the halo pulses which could also be asymmetric, as suggested by simulations. The exact parameters of the halo may differ for different $\mathrm{X}$-ray photon energies and beamline settings as well as depending on the performance of the slicing source.

\subsection{Photon energy and polarization}

While the sliced photons are emitted from on-axis electrons with lower energy $(-1 \%)$ at $\sim 0.2 \%$ width, the halo photons can arrive in the beamline also from all electrons within a maximum spread of $-1 \% \ldots+1 \%$ since after a few turns they pass the radiator traveling on randomly distributed tracks. Hence, the energy position of the undulator harmonic is much wider and shifted in energy by a few $\mathrm{eV}$ with respect to one of the sliced photons, which gives one another handle to spectrally separate halo photons.

Further complications arise from undulator operation in elliptical mode, which is mandatory for XMCD experiments. Since the halo can arrive in this case at the detector also from a larger angular range $(>1 / \gamma=0.3 \mathrm{mrad}$ at BESSY II), the degree of circular polarization of halo photons is reduced or even flipped to the other helicity by compensation effects between far off-axis and on-axis photons (Khan et al., 2006).

In order to characterize the polarization properties of the halo radiation, we performed a transmission XMCD experiment on a $\mathrm{Gd}_{18} \mathrm{Fe}_{82}(30 \mathrm{~nm})$ sample deposited on a $500 \mathrm{~nm} \mathrm{Al}$ foil at the $\mathrm{Fe} L_{3}$-edge $(\sim 708 \mathrm{eV})$. Here we purposely used a rather small slicing-to-halo ratio of $\beta=0.67$ for a transmission experiment in order to demonstrate an experimental worst case scenario and to be comparable with the resonant diffraction experiment in the former subsection. The experimental results are shown in Fig. 8(a) as symbols and the solid lines represent fits using equations (11) and (13).

One can directly determine the XMCD amplitudes $\alpha_{\mathrm{S}}=$ -0.023 for the slicing and $\alpha_{\mathrm{H}}=0.015$ for the halo data from the unpumped XMCD signals $\chi_{0}=\chi(t \ll 0)$ at early delays. The difference in sign and amplitude of the extracted halo and slicing XMCD amplitudes arises from the above-mentioned fact that the halo radiation entering the beamline is partly off the optical axis of the undulator and therefore shows opposite sign and smaller degree of helicity with respect to the sliced X-rays.

It is worth investigating the effect of these different degrees of X-ray polarization on the measured sample dynamics. For this, we describe the photo-induced magnetization dynamics of the sample by a single exponential decay,

$$
m(t)=A H(t)[\exp (-t / \tau)-1]+1
$$

with $\tau$ as time constant and $A$ as amplitude. From the fit of the extracted slicing data, $\chi_{\mathrm{S}}(t)$, using equation (11), we derive $\tau=0.3$ ps and $A_{\mathrm{S}}=0.82$. In order to fit the halo data, $\chi_{\mathrm{H}}(t)$, we

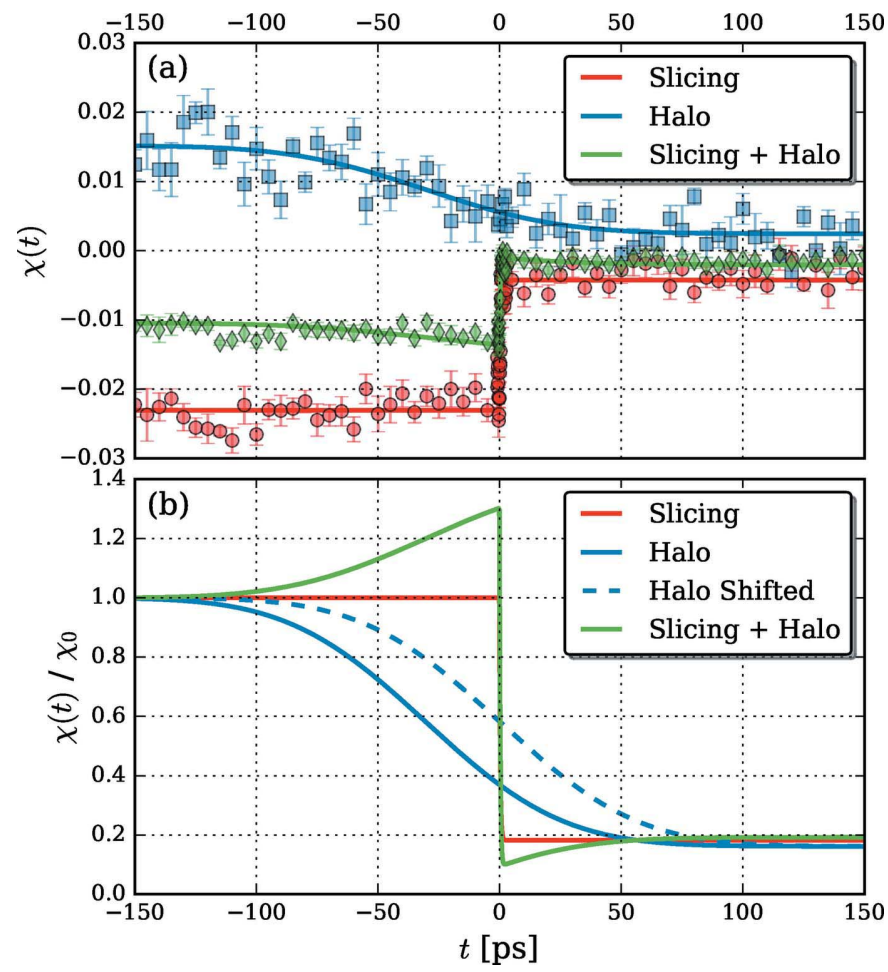

Figure 8

(a) Time-resolved XMCD measurement at the Fe $L_{3}$-edge $(708 \mathrm{eV})$ in transmission on a $\mathrm{Gd}_{0.18} \mathrm{Fe}_{0.82}$ sample. The symbols represent the experimental data and the solid lines the corresponding fits; see text. The pure slicing contribution (red) is determined by subtracting the halo (blue) from the original slicing + halo (green) data. The slicing fraction of the signal is $\beta=0.67$. Note that the subtraction is performed with absolute and not relative data. $(b)$ XMCD fits from $(a)$ on a relative scale for better comparison. The blue dashed line represents the halo fit but shifted to the same time zero as for the slicing.

fix the fit result from above, but use the demagnetization amplitude $A$, as well as the width and arrival time of the halo pulse $g_{\mathrm{H}}(t)$, as free parameters. From the fit of the halo XMCD we again derive a prolonged halo pulse width of $\sigma_{\mathrm{H}}^{\mathrm{FWHM}}=$ $107 \mathrm{ps}$ and a delay with respect to the slicing pulse of $t_{0, \mathrm{H}}=$ 30 ps. Fig. $8(b)$ highlights the latter fact by comparing the XMCD fits from Fig. $8(a)$ on a relative scale for all three contributions also showing the halo XMCD shifted in delay to match the slicing pulses. The amplitude of the demagnetization for the halo is rather similar to slicing with $A_{\mathrm{H}}=0.84$ which points to a rather homogeneous excitation of the sample. The fit for the sliced + halo X-rays is derived from applying the fit results of just the sliced and halo X-rays from above to equation (13), using $\beta=0.67$ as slicing fraction derived from equation (15).

The above-presented experimental data demonstrate the complexity of the dynamic halo background especially for time-resolved XMCD experiments. Without proper simultaneous measurement of the dynamic halo a quantitative evaluation can be afflicted with errors. These errors are associated with the individual temporal and spatial properties of the halo contribution, and its opposite helicity with respect to the pure sliced X-rays. These potential errors are the subject of the following section. 


\section{Error estimation}

The experimental characterization presented in the last section revealed that the spatial and temporal profile as well as the polarization are the most important sources of systematic errors for the correct halo treatment in femtosecond pumpprobe experiments at the FemtoSpeX facility at BESSY II. Although the new triggering scheme at the beamline allows for direct measurement of the halo contributions to any timeresolved experiment it leads to a doubled accumulation time. Its application therefore requires a thorough comparison with analytical methods for proper halo background removal. For this purpose we simulate error-free experimental data of a transmission XMCD experiment assuming a single exponential decay of the magnetization $m(t)$ [see equation (17)]. For this generic case we set the experimental parameters according to Table 1 and the slicing portion to a worst case value of $\beta=0.6$, similar to the data presented in Fig. 8(a), and a best case value of $\beta=0.9$. We used equations (11) and (13) for calculating the generic data [see Figs. $9(a)$ and $9(b)$ for only the worst case scenario].

For fitting the slicing + halo XMCD we discuss two distinct cases: (i) we completely neglect the halo background using only equation (11), fixing the arrival time and width of the slicing pulse according to Table 1 and use $A, \alpha$ and $\tau$ as free parameters of the fit; (ii) a simplified approach where we apply equation (13) as a fit function, which was also used to simulate the data. For the latter approach not all halo characteristics are known at any time of an experiment, in order to save precious beam time. Instead, one estimates/simplifies the halo

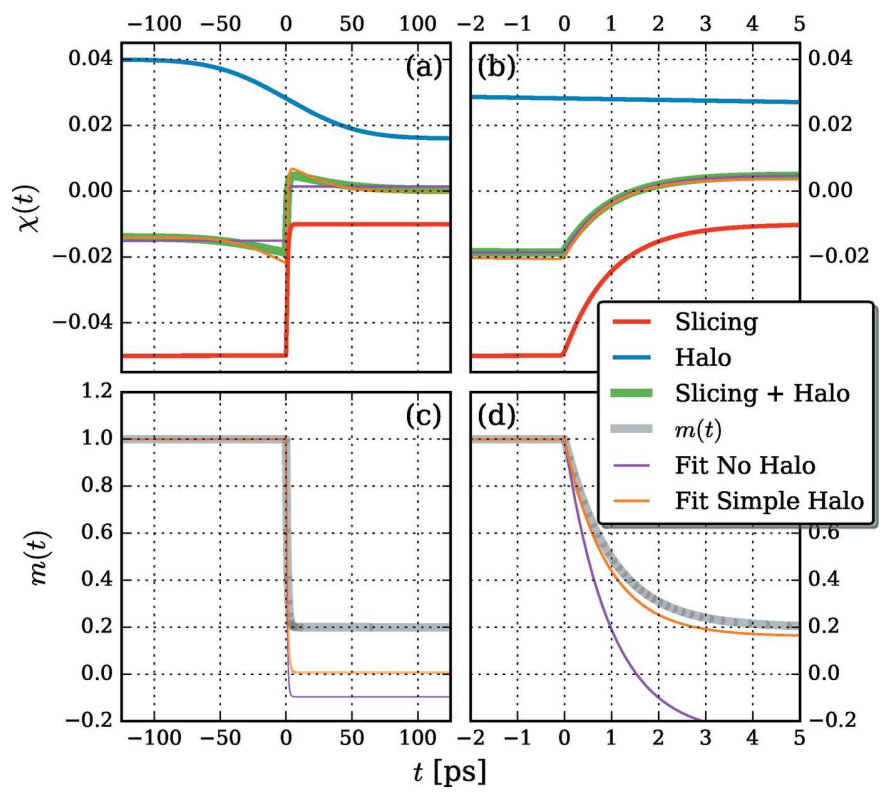

Figure 9

$(a, b)$ Simulated XMCD data (thick solid lines) on a long and short delay range for the worst case scenario of $\beta=0.6$. The thin solid lines represent the fits neglecting any halo contribution and a simplified halo contribution, respectively. $(c, d)$ Initial magnetization dynamics $m(t)$ and the results derived from the fits of the according upper panels $(a)$ and $(b)$. Note that panels $(b)$ and $(d)$ are not a zoom-in, instead the fit routines are applied only to the shown subset of the simulated slicing + halo XMCD data shown in $(a)$.
Table 1

Experimental parameters for the simulation of generic XMCD data.

\begin{tabular}{llllll}
\hline & $t_{0}(\mathrm{ps})$ & $\sigma^{\text {FWHM }}(\mathrm{ps})$ & $A$ & $\alpha$ & $\tau(\mathrm{ps})$ \\
\hline Slicing & 0 & 0.1 & 0.8 & -0.05 & 1 \\
Halo & 0 & 100 & 0.6 & +0.04 & 1 \\
\hline
\end{tabular}

Table 2

Fitted values and relative errors for the worst case fits $(\beta=0.6)$ shown in Fig. 9 and best case fits $(\beta=0.9)$; plots not shown.

\begin{tabular}{lllllll}
\hline Range (ps) & $\alpha_{\mathrm{S}}$ & $\Delta \alpha_{\mathrm{S}} / \alpha_{\mathrm{S}}$ & $A$ & $\Delta A / A$ & $\tau(\mathrm{ps})$ & $\Delta \tau / \tau$ \\
\hline Input model & -0.05 & - & 0.8 & - & 1.0 & - \\
$\beta=0.6$, no halo fit & & & & & \\
{$[-2,5]$} & -0.018 & $-64 \%$ & 1.26 & $+58 \%$ & 0.97 & $-3 \%$ \\
{$[-125,125]$} & -0.015 & $-70 \%$ & 1.10 & $+38 \%$ & 0.75 & $-25 \%$ \\
$\beta=0.6$, simple halo fit & & & & & \\
{$[-2,5]$} & - & - & 0.84 & $+5 \%$ & 0.83 & $-17 \%$ \\
{$[-125,125]$} & - & - & 1.00 & $+25 \%$ & 1.25 & $+25 \%$ \\
$\beta=0.9$, no halo fit & & & & & \\
{$[-2,5]$} & -0.042 & $-16 \%$ & 0.85 & $+6 \%$ & 0.98 & $-2 \%$ \\
{$[-125,125]$} & -0.041 & $-18 \%$ & 0.83 & $+4 \%$ & 0.95 & $-5 \%$ \\
$\beta=0.9$, simple halo fit & & & & & \\
{$[-2,5]$} & - & - & 0.80 & $\pm 0 \%$ & 0.94 & $-6 \%$ \\
{$[-125,125]$} & - & - & 0.82 & $+3 \%$ & 1.00 & $0 \%$ \\
\hline
\end{tabular}

characteristics for the data evaluation. Here we assume a fixed halo pulse width similar to the single bunch width of $\sigma_{\mathrm{H}}^{\mathrm{FWHM}}=$ 75 ps. Moreover, we use the same amplitude $A$ and decay constant $\tau$ in the fit as free parameters. For the slicing portion $\beta$ as well as the halo and slicing XMCD amplitudes, $\alpha_{\mathrm{H}}$ and $\alpha_{\mathrm{S}}$, we use the exact values from Table 1 as fixed fit parameters, since they can be directly derived from equation (15) and from the unpumped signals, respectively.

We apply the two fit procedures for a large delay range of the slicing + halo XMCD $(-125<t<125 \mathrm{ps})$, see Fig. 9(a), and for a short (more realistic) delay range $(-\tau<t<5 \tau$, with $\tau=1 \mathrm{ps}$ ), see Fig. $9(b)$. We always use the input parameters of the simulated data as initial values for the according fit parameters. The derived magnetization dynamics $m(t)$ are then plotted in the corresponding lower panels of Fig. 9 for only the worst case scenario of $\beta=0.6$ and the fit results are listed in Table 2 for both cases.

For the worst case scenario, $\beta=0.6$, Fig. $9(b)$ shows that for short time scales the halo background (blue line) can be simplified as a delay-independent background contribution to the overall signal. For this case, the correct determination of the exponential decay constant $\tau$ of the demagnetization dynamics $m(t)$ is nearly unaffected by the halo background if $\tau \ll \sigma_{\mathrm{H}}$. The best result for $\tau$ is actually achieved by completely neglecting the halo and fitting only on a short delay range. Neglecting the halo in a long delay range fit gives worse results for $\tau$, since the simple fit function cannot reflect the more complex time dependence of the input data. Not surprisingly, for the chosen input parameters, both fit models on both delay ranges fail to extract the demagnetization amplitude $A$ and the absolute XMCD amplitude $\alpha$ with 
satisfying precision. An analysis neglecting the halo even results in a sign change of the XMCD signal after $\sim 1.5 \mathrm{ps}$, see Figs. $9(c)-9(d)$, erroneously suggesting a switching of the sample magnetization $m(t)$. For the best case scenario of $\beta=$ 0.9 the results in Table 2 confirm the small errors made for the decay constant $\tau$ for all four cases. However, the determination of the absolute XMCD amplitude $\alpha$ is still error prone when neglecting the halo contribution to the signal completely. In any case, a careful estimation of the halo contribution to the overall signal and to the extracted fitting parameters should be conducted before applying the here-presented experimental or analytical halo correction routines.

\section{Conclusion}

In this contribution we have given a thorough description of the so-called halo background inherent at the FemtoSpeX facility at BESSY II. We implemented a dedicated laser triggering scheme to easily access the halo background for any time-resolved experiment. With this technique we characterized the spatial, temporal and polarization properties of the halo radiation in great detail. The triggering scheme allows for exact determination and removal of the halo contribution from the femtosecond time-resolved X-ray traces. This makes a precise quantitative evaluation of the data possible. Based on our measurements we estimated the relative errors made in a worst and best case generic XMCD pump-probe experiment regarding the slicing-to-halo ratio. For the best case scenario, realised for example for transmission experiments with high photon flux available on the detector, the halo background only influences the correct determination of the absolute XMCD amplitude, when fully neglecting the halo in the data analysis. All other relevant fit parameters can be deduced within acceptable error limits of $\sim \pm 5 \%$. In a worst case scenario, for example for diffraction/reflection experiments with low photon flux on the detector, even a simplified halo fit model leads to significant errors for the amplitudes of the XMCD, the amount of demagnetization, and even the demagnetization time constant. For such slicing-to-halo ratios a thorough error estimation for each sample and any experimental condition has to be carried out. However, the described analytic treatment of the experimental data as well as the presented direct experimental access to the halo background lead to a significantly improved quantitative precision of the FemtoSpeX facility at BESSY II.

\section{Acknowledgements}

We thank Harmut Zabel for providing the dysprosium sample. DS acknowledges the Helmholtz Association for funding via the Helmholtz Postdoc Programme PD-142. Funding from European Union through FEMTOSPIN program is gratefully acknowledged. This work was further supported by the PeterPaul-Ewald Fellowship of the Volkswagen Stiftung.

\section{References}

Beaud, P., Johnson, S., Streun, A., Abela, R., Abramsohn, D., Grolimund, D., Krasniqi, F., Schmidt, T., Schlott, V. \& Ingold, G. (2007). Phys. Rev. Lett. 99, 174801.

Beaud, P., Johnson, S., Vorobeva, E., Staub, U., De Souza, R. A., Milne, C., Jia, Q. \& Ingold, G. (2009). Phys. Rev. Lett. 103, 155702.

Beye, M., Krupin, O., Hays, G., Reid, A. H., Rupp, D., de Jong, S., Lee, S., Lee, W. S., Chuang, Y. D., Coffee, R., Cryan, J. P., Glownia, J. M., Föhlisch, A., Holmes, M. R., Fry, A. R., White, W. E., Bostedt, C., Scherz, A. O., Durr, H. A. \& Schlotter, W. F. (2012). Appl. Phys. Lett. 100, 121108.

Brzhezinskaya, M., Firsov, A., Holldack, K., Kachel, T., Mitzner, R., Pontius, N., Schmidt, J.-S., Sperling, M., Stamm, C., Föhlisch, A. \& Erko, A. (2013). J. Synchrotron Rad. 20, 522-530.

Carva, K., Legut, D. \& Oppeneer, P. M. (2009). Europhys. Lett. 86, 57002.

Emma, P., Akre, R., Arthur, J., Bionta, R., Bostedt, C., Bozek, J., Brachmann, A., Bucksbaum, P., Coffee, R., Decker, F.-J., Ding, Y., Dowell, D., Edstrom, S., Fisher, A., Frisch, J., Gilevich, S., Hastings, J., Hays, G., Hering, P., Huang, Z., Iverson, R., Loos, H., Messerschmidt, M., Miahnahri, a, Moeller, S., Nuhn, H.-D., Pile, G., Ratner, D., Rzepiela, J., Schultz, D., Smith, T., Stefan, P., Tompkins, H., Turner, J., Welch, J., White, W., Wu, J., Yocky, G. \& Galayda, J. (2010). Nat. Photon. 4, 641-647.

Eschenlohr, A., Battiato, M., Maldonado, P., Pontius, N., Kachel, T., Holldack, K., Mitzner, R., Föhlisch, A., Oppeneer, P. M. \& Stamm, C. (2013). Nat. Mater. 12, 332-336.

Harmand, M., Coffee, R., Bionta, M. R., Chollet, M., French, D., Zhu, D., Fritz, D. M., Lemke, H. T., Medvedev, N., Ziaja, B., Toleikis, S. \& Cammarata, M. (2013). Nat. Photon., 7, 215-218.

Holldack, K., Bahrdt, J., Balzer, A., Bovensiepen, U., Brzhezinskaya, M., Erko, A., Eschenlohr, A., Follath, R., Firsov, A., Frentrup, W., Le Guyader, L., Kachel, T., Kuske, P., Mitzner, R., Müller, R., Pontius, N., Quast, T., Radu, I., Schmidt, J.-S., Schüßler-Langeheine, C., Sperling, M., Stamm, C., Trabant, C. \& Föhlisch, A. (2014). J. Synchrotron Rad. 21, 1090-1104.

Holldack, K., Khan, S., Mitzner, R. \& Quast, T. (2006). Phys. Rev. Lett. 96, 054801.

Holldack, K., Pontius, N., Schierle, E., Kachel, T., Soltwisch, V., Mitzner, R., Quast, T., Springholz, G. \& Weschke, E. (2010). Appl. Phys. Lett. 97, 062502.

Khan, S., Holldack, K., Kachel, T., Mitzner, R. \& Quast, T. (2006). Phys. Rev. Lett. 97, 074801.

Kubacka, T., Johnson, J. A., Hoffmann, M. C., Vicario, C., de Jong, S., Beaud, P., Grubel, S., Huang, S.-W., Huber, L., Patthey, L., Chuang, Y. D., Turner, J. J., Dakovski, G. L., Lee, W. S., Minitti, M. P., Schlotter, W., Moore, R. G., Hauri, C. P., Koohpayeh, S. M., Scagnoli, V., Ingold, G., Johnson, S. L. \& Staub, U. (2014). Science, 343, 1333-1336.

Kuske, P. (2011). Proceedings of the 2011 International Particle Accelerator Conference, 4-9 September 2011, San Sebastián, Spain, pp. 3397-3399.

Ott, H., Schüßler-Langeheine, C., Schierle, E., Grigoriev, A. Yu., Leiner, V., Zabel, H., Kaindl, G. \& Weschke, E. (2006). Phys. Rev. B, 74, 094412.

Patterson, B. D., Abela, R., Braun, H.-H., Flechsig, U., Ganter, R., Kim, Y., Kirk, E., Oppelt, A., Pedrozzi, M., Reiche, S., Rivkin, L., Schmidt, T., Schmitt, B., Strocov, V. N., Tsujino, S. \& Wrulich, A. F. (2010). New J. Phys. 12, 035012.

Prigent, P., Hollander, P., Labat, M., Couprie, M. E., Marlats, J. L., Laulhé, C., Luning, J., Moreno, T., Morin, P., Nadji, A., Polack, F., Ravy, S., Silly, M. \& Sirotti, F. (2013). J. Phys. Conf. Ser. 425, 072022.

Radu, I., Vahaplar, K., Stamm, C., Kachel, T., Pontius, N., Dürr, H. A., Ostler, T. A., Barker, J., Evans, R. F. L., Chantrell, R. W., 
Tsukamoto, A., Itoh, A., Kirilyuk, A., Rasing, T. \& Kimel, A. V. (2011). Nature (London), 472, 205-208.

Schäfers, F. (2008). Modern Develepoments in X-ray Neutron Optics, pp. 9-41. Berlin/Heidelberg: Springer.

Scheer, M. (2012). Proceedings of the 11th International Computational Accelerator Physics Conference (ICAP-2012), RostockWarnemünde, Germany, p. 88.

Schoenlein, R. W., Chattopadhyay, S., Chong, H., Glover, T. E., Heimann, P., Shank, C. V., Zholents, A. \& Zolotorev, M. (2000). Science, 287, 2237-2240.
Stamm, C., Kachel, T., Pontius, N., Mitzner, R., Quast, T., Holldack, K., Khan, S., Lupulescu, C., Aziz, E. F., Wietstruk, M., Dürr, H. A. \& Eberhardt, W. (2007). Nat. Mater. 6, 740-743.

Stöhr, J. \& Siegmann, H. C. (2006). Magnetism: From Fundamentals to Nanoscale Dynamics, Springer Series in Solid-State Sciences, Vol. 152. Heidelberg: Springer.

Streun, A. (2010). Halo background in laser beam slicing experiments at SLS and SOLEIL. Technical Report SLS-TME-TA-2010-0320, 10 March 2010. Paul Scherrer Institut, Villigen, Switzerland.

Zholents, A. \& Zolotorev, M. S. (1996). Phys. Rev. Lett. 76, 912-915. 